\title{
ROJ
}

\section{Prognostic value of FDG PET/CT during radiotherapy in head and neck cancer patients}

\author{
Suzy Kim, MD, PhD ${ }^{1 *}$, Sowon Oh, MD, PhD ${ }^{2 *}$, Jin Soo Kim, MD, PhD ${ }^{3}$, \\ Yu Kyeong Kim, MD, PhD², Kwang Hyun Kim, MD, PhD ${ }^{4}$, Do Hoon Oh, MD ${ }^{5}$ Dong-Han Lee, $\mathrm{MD}^{6}$, \\ Woo-Jin Jeong, MD, $\mathrm{PhD}^{6}$, Young Ho Jung, MD, $\mathrm{PhD}^{6}$ \\ Departments of ${ }^{1}$ Radiation Oncology, ${ }^{2}$ Nuclear Medicine, ${ }^{3}$ Internal Medicine, and ${ }^{4}$ Otolaryngology, \\ Seoul National University Boramae Medical Center, Seoul; \\ ${ }^{5}$ Department of Radiation Oncology, Chung-Ang University Hostpial, Seoul; \\ ${ }^{6}$ Department of Otorhinolaryngology-Head and Neck Surgery, Seoul National University Bundang Hospital, Seongnam, Korea
}

\begin{abstract}
Purpose: To evaluate the prognostic value of ${ }^{18} \mathrm{~F}$-fluorodeoxyglucose positron-emission tomography (FDG PET) with computed tomography (CT) before and during radiotherapy (RT) in patients with head and neck cancer.

Methods: Twenty patients with primary head and neck squamous cell carcinoma were enrolled in this study, of whom 6 had oropharyngeal cancer, 10 had hypopharyngeal cancer, and 4 had laryngeal cancer. Fifteen patients received concurrent cisplatin and 2 received concurrent cetuximab chemotherapy. FDG PET/CT was performed before RT and in the 4th week of RT. The parameters of maximum standardized uptake value, metabolic tumor volume, and total lesion glycolysis (TLG) of the primary tumor were measured, and the prognostic significance of each was analyzed with the Cox proportional hazards model.

Results: Higher TLG (>19.0) on FDG PET/CT during RT was a poor prognostic factor for overall survival (OS) $(p=0.001)$ and progression-free survival (PFS) $(p=0.007)$. In the multivariate analysis, TLG during RT as a continuous variable was significantly associated with OS and PFS rate ( $p=0.023$ and $p=0.016$, respectively). Tumor response worse than partial remission at 1 month after RT was another independent prognostic factor for PFS ( $p=0.024)$.

Conclusions: Higher TLG of the primary tumor on FDG PET/CT during RT was a poor prognostic factor for OS and PFS in patients with head and neck cancer.
\end{abstract}

Keywords: Head and neck neoplasms, Positron-emission tomography, Radiotherapy, Prognostic factor

\section{Introduction}

Cancers of the head and neck are relatively rare, accounting for about $3 \%$ of all malignancies [1]. Nearly $60 \%$ of patients with head and neck cancer present with locally advanced but non-metastatic disease. For locally advanced or unresectable head and neck cancer, radiotherapy (RT) with or without chemotherapy plays an important role. A meta-analysis of

Received 22 December 2017, Revised 31 January 2018, Accepted 14 March 2018.

Correspondence: Young Ho Jung, MD, PhD, Department of Otorhinolaryngology-Head and Neck Surgery, Seoul National University Bundang Hospital, 82 Gumi-ro 173beon-gil, Bundang-gu, Seongnam 13620, Korea. Tel: +82-31-787-7413, Fax: +8231-787-4057, E-mail: entist@naver.com

*Both authors contributed equally to this work.

(c) This is an Open Access article distributed under the terms of the Creative Commons Attribution Non-Commercial License (http://creativecommons.org/ licenses/by-nc/4.0/) which permits unrestricted non-commercial use, distribution, and reproduction in any medium, provided the original work is properly cited.

www.e-roj.org 
chemotherapy in head and neck cancer patients with locally advanced disease revealed that concurrent chemoradiotherapy (CCRT) improved 5-year overall survival (OS) compared with RT alone [2]. Cisplatin is the systemic agent of choice in combination with RT, but cetuximab, an anti-epidermal growth factor receptor 1 monoclonal antibody, can also be used for treatment $[3,4]$.

Although CCRT improves survival, it increases the rate of acute or chronic toxicities compared with RT alone. A large number of head and neck cancer patients receiving CCRT suffer from acute toxicities, and some experience toxicityrelated treatment delay or hospitalization [5]. After receiving CCRT for locally advanced head and neck cancer, locoregional recurrence rates are 30\%-50\% and distant metastasis rates range from $15 \%$ to $20 \%[6,7]$. The likelihood of successful salvage treatment for recurrent disease is low. Therefore, it is necessary to determine which subgroup of head and neck cancer patients do not benefit from CCRT or RT and have a high probability of disease relapse. The early identification of these patients would enable the selection of appropriate treatment.

18F-fluorodeoxyglucose positron-emission tomography (FDG PET) combined with computed tomography (CT) has higher sensitivity than FDG PET or CT alone for detecting primary tumors in the head and neck $[8,9]$. It is not only useful for staging and RT planning of head and neck cancer, some of its metabolic parameters are known to have prognostic value as well $[10,11]$. Recent studies have assessed metabolic changes in FDG PET/CT during treatment as a method for predicting tumor response to therapy and survival outcomes $[12,13]$.

There are many reports on the prognostic value of FDG PET/CT before or after RT. However, relatively little data are available on the predictive value of FDG PET/CT during RT for head and neck cancer. The aim of this study was to perform quantitative evaluation of the ability of FDG PET/CT parameters to predict the treatment outcomes of head and neck cancer patients, in scanning performed before and during RT.

\section{Materials and Methods}

\section{Patients and treatment methods}

Enrolled in this prospective study were 20 patients with squamous cell carcinoma of the head and neck who were treated at Seoul National University Boramae Medical Center between March 2012 and March 2014. Head and neck cancer patients who received definitive RT or chemoradiotherapy were included in this study. Patients who had early glottic cancer, nasopharyngeal cancer, distant metastasis, previous RT, or previous definitive surgery were excluded. This study protocol was approved by our institutional review board. The tumor location was hypopharyngeal in 10, oropharyngeal in 6, and laryngeal in 4 patients. Histologically, all lesions were squamous cell carcinoma.

All patients were treated with intensity-modulated RT with a simultaneous integrated boost technique. All of the primary and nodal gross tumor volume, with a $10-\mathrm{mm}$ margin, was defined as the clinical target volume 1 (CTV1); CTV2 included high-risk nodal regions and a $5-\mathrm{mm}$ margin additional to the CTV1; CTV3 included the low-risk nodal volume. A radiation dose of $70 \mathrm{~Gy}$ in 35 fractions (2 Gy/fraction) was prescribed to CTV1, 63 Gy in 35 fractions (1.8 Gy/fraction) was prescribed to CTV2, and $56 \mathrm{~Gy}$ in 35 fractions (1.6 Gy/fraction) was prescribed to CTV3.

Seventeen of 20 patients (85\%) received concurrent chemotherapy. Fifteen patients received concurrent weekly cisplatin chemotherapy (cisplatin $35 \mathrm{mg} / \mathrm{m}^{2}$ intravenous [IV] weekly) and two patients received concurrent cetuximab (cetuximab $400 \mathrm{mg} / \mathrm{m}^{2}$ IV on 7 days before the start of RT, and $250 \mathrm{mg} / \mathrm{m}^{2}$ IV weekly during RT). Two patients with stage T2NO and T3NO hypopharyngeal cancer and one patient with clinically node-negative subglottic cancer did not receive concurrent chemotherapy.

Five patients received three cycles of neoadjuvant chemotherapy before CCRT, four of whom were treated with a combined regimen of docetaxel $\left(70 \mathrm{mg} / \mathrm{m}^{2} \mathrm{IV}\right.$ on D1), cisplatin (40 mg/m $/ \mathrm{m}^{2}$ IV on D2, D3), and 5-fluorouracil (400 mg/m $/ \mathrm{m}^{2}$ on D1-D3) every 3 weeks. The remaining patient was treated with paclitaxel $\left(230 \mathrm{mg} / \mathrm{m}^{2} \mathrm{IV}\right.$ on D1) and cisplatin $\left(60 \mathrm{mg} / \mathrm{m}^{2} \mathrm{IV}\right.$ on D1) every 3 weeks.

The clinical response of the tumor to RT was evaluated on CT performed 1 month after completion of RT. At 3 months after RT, follow-up CT and PET/CT were performed and salvage surgical treatments were considered for any residual or recurrent locoregional lesions. Three patients with suspected residual lymph nodes underwent salvage neck dissection at 3, 5, and 9 months after RT, respectively. One of them didn't have any metastatic lymph node at surgical specimen and the others were successfully salvaged. Another three patients received salvage total laryngectomy with or without neck dissection at 3 months, 3 months and 4 months after RT, respectively. Two of them didn't have any evidence of disease after surgery but the other one patient expired at 3 months after surgery because of the bleeding from common carotid 
artery pseudoaneurysm.

\section{FDG PET/CT imaging and measurement of PET/CT parameters}

FDG PET/CT images were acquired in all 20 patients, using a Gemini TF scanner (Philips Healthcare, Cleveland, OH, USA) before and during RT. The timing of scanning during RT ranged from the 3rd to 4th week of RT (median, 26 days from the start of RT). The PET/CT scanning methods used were as previously described [14]. The acquired PET/CT images were transferred to a dedicated workstation and analyzed using the vendor-provided software (The Extended Brilliance Workspace with Fusion Viewer, Philips Healthcare). The software of the workstation provided automatically delineated volume-ofinterest (VOI) over the tumor using a threshold of 50\% of maximum standardized uptake value $\left(S \cup V_{\text {max }}\right)$ [15]. Metabolic tumor volume (MTV) was defined as those voxels having an

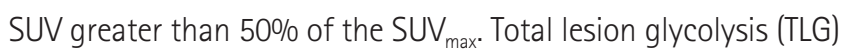
was calculated by multiplying MTV by the mean standardized uptake value $\left(S U V_{\text {mean }}\right)$. SUV, MTV, and TLG were measured at the primary tumor site. There were 3 patients whose metabolic parameters were unmeasurable at the primary tumor site after neoadjuvant chemotherapy, and their PET/CT parameters were measured at the metastatic nodal sites. If the tumor could not be distinguished from the background, MTV was set as a single voxel with a volume of $0.1 \mathrm{~cm}^{3}$. SUV was assigned with a default value of 1.0, which was the minimum value [16].

\section{Statistical analyses}

Receiver operating characteristic (ROC) curve analysis was used to identify the optimal cut-off value for continuous PET/ CT parameters, as the maximal point of the sum of sensitivity and specificity. All patients were divided into two subgroups for good or poor outcome in terms of OS or progression-free survival (PFS) for each clinical variable and PET/CT parameter. OS was defined as the time interval between the date of any first treatment and the date of death or last follow-up. PFS was defined as the time from the date of any first treatment to the date of locoregional or distant recurrence. Death without documented recurrence was censored at the time of death. The Kaplan-Meier method was used to calculate the 3-year OS and PFS rates. In univariate analysis, the log-rank test was used to compare the clinical variables and PET/CT parameters. In multivariate analysis, the Cox proportional hazard model was used to identify independent prognostic factors of OS and PFS. Paired t-test was used to compare the PET/CT parameters before and during RT. Statistical significance was defined as a $p$-value $<0.05$ (two-sided). All statistical analyses were performed using SPSS version 20.0 software (IBM Corporation, Armonk, NY, USA).

\section{Results}

\section{Clinical characteristics and FDG PET/CT parameters}

The clinical characteristics of the patients are summarized in Table 1. There were $4(20 \%)$ patients with radiological T1 or T2 tumors and 16 (80\%) with T3 or T4. Nine patients (45\%) were clinical N0 stage, and 11 (55\%) were N1 or N2 stage. The clinical response of the tumor to RT was evaluated on neck $C T$,

Table 1. Patient characteristics

\begin{tabular}{|c|c|}
\hline Characteristic & Value \\
\hline Age (yr) & $60.5(45-82)$ \\
\hline \multicolumn{2}{|l|}{ Sex } \\
\hline Female & $2(10)$ \\
\hline Male & $18(90)$ \\
\hline \multicolumn{2}{|l|}{ Primary site } \\
\hline Hypopharynx & $10(50)$ \\
\hline Oropharynx & $6(30)$ \\
\hline Larynx & $4(20)$ \\
\hline \multicolumn{2}{|l|}{ ECOG performance status } \\
\hline 0 & $2(10)$ \\
\hline 1 & $17(85)$ \\
\hline 2 & $1(5)$ \\
\hline \multicolumn{2}{|l|}{ T stage } \\
\hline $\mathrm{T} 1$ & $2(10)$ \\
\hline $\mathrm{T} 2$ & $2(10)$ \\
\hline T3 & $9(45)$ \\
\hline $\mathrm{T} 4$ & $7(35)$ \\
\hline \multicolumn{2}{|l|}{ N stage } \\
\hline No & $9(45)$ \\
\hline N1 & $0(0)$ \\
\hline N2 & $10(50)$ \\
\hline N3 & $1(5)$ \\
\hline \multicolumn{2}{|l|}{ Stage } \\
\hline$\|$ & $2(10)$ \\
\hline III & $4(20)$ \\
\hline IV & $14(70)$ \\
\hline \multicolumn{2}{|l|}{ Neoadjuvant chemotherapy } \\
\hline No & $15(75)$ \\
\hline Docetaxel + cisplatin + 5-FU & $4(20)$ \\
\hline Paclitaxel + cisplatin & $1(5)$ \\
\hline \multicolumn{2}{|l|}{ Concurrent chemotherapy } \\
\hline No & $3(15)$ \\
\hline Cisplatin & $15(75)$ \\
\hline Cetuximab & $2(10)$ \\
\hline
\end{tabular}

Values are presented as median (range) or number (\%). ECOG, Eastern Cooperative Oncology Group; 5-FU, 5-fluorouracil. 
1 month after the completion of RT. According to the revised RECIST criteria (v.1.1), 4 patients (20\%) showed a complete response (CR), 11 patients (55\%) had a partial response (PR), and 5 (25\%) had stable disease (SD). In the FDG PET/CT before $R T$, the SUV ${ }_{\max }$ of the primary tumors ranged from 2.0 to 15.8 $\mathrm{g} / \mathrm{mL}$, with a mean value of $6.9 \pm 3.8 \mathrm{~g} / \mathrm{mL}$. During $R T$, the SUV $_{\text {max }}$ significantly decreased and ranged between 1.4 and $10.2 \mathrm{~g} / \mathrm{mL}$, with a mean value of $4.2 \pm 2.5 \mathrm{~g} / \mathrm{mL}$. The mean value of SUV $V_{\text {mean }}$ before RT was $4.7 \pm 2.6 \mathrm{~g} / \mathrm{mL}$ (range, 1.0 to $10.5 \mathrm{~g} / \mathrm{mL}$ ), and that during RT also significantly decreased and was $2.7 \pm 1.8 \mathrm{~g} / \mathrm{mL}$ (range, 1.0 to $7.0 \mathrm{~g} / \mathrm{mL}$ ). Table 2 shows the FDG PET/CT parameters before and during RT.

\section{Survival analysis}

The median follow-up time was 49 months (range, 42 to 60 months). Fifteen patients (75\%) were alive at last follow-up. The 3-year OS rate of all 20 patients was $80.0 \%$ and the 3 -year PFS was $60.0 \%$.

Before RT, the optimal cut-offs for PET/CT parameters derived from the ROC curves were: SUVmax (preSUV $\left.V_{\text {max }}\right)=8.0$ $\mathrm{g} / \mathrm{mL}$ (area under the ROC curve $[A U C]=0.57, p=0.631$ ), MTV (preMTV) $=5.5 \mathrm{~cm}^{3}(\mathrm{AUC}=0.71, \mathrm{p}=0.176)$, and TLG (preTLG) $=39 \mathrm{~g}(\mathrm{AUC}=0.68, p=0.239)$. The 3 -year OS rate was higher in patients with preTLG $<39 \mathrm{~g}$ than in those with preTLG $>39 \mathrm{~g}$ ( $88 \%$ vs. $50 \% ; p=0.004$ ).

During RT, the optimal cut-offs for PET/CT parameters were: SUVmax $\left(\operatorname{midSUV}_{\max }\right)=3.5 \mathrm{~g} / \mathrm{mL}(A U C=0.74, p=0.116)$, MTV $($ midMTV $)=4.5 \mathrm{~cm}^{3}(\mathrm{AUC}=0.81, \mathrm{p}=0.040)$, and TLG (midTLG) $=19.0 \mathrm{~g}$ ( $\mathrm{AUC}=0.91, \mathrm{p}=0.008$ ). The 3 -year OS rate was higher in patients with midTLG $<19.0 \mathrm{~g}$ than in those with midTLG $>19.0 \mathrm{~g}(100 \%$ vs. $50 \% ; p=0.001)$. OS rate was lower in patients with a higher midMTV $\left(>4.5 \mathrm{~cm}^{3}\right)$ than in those with a lower midMTV $\left(<4.5 \mathrm{~cm}^{3}\right)$ (3-year rate, $64 \%$ vs. $100 \%$; $p$ $=0.022$ ).

Patients with tumor response better than PR after RT had a higher PFS rate than those without tumor response to RT (SD)
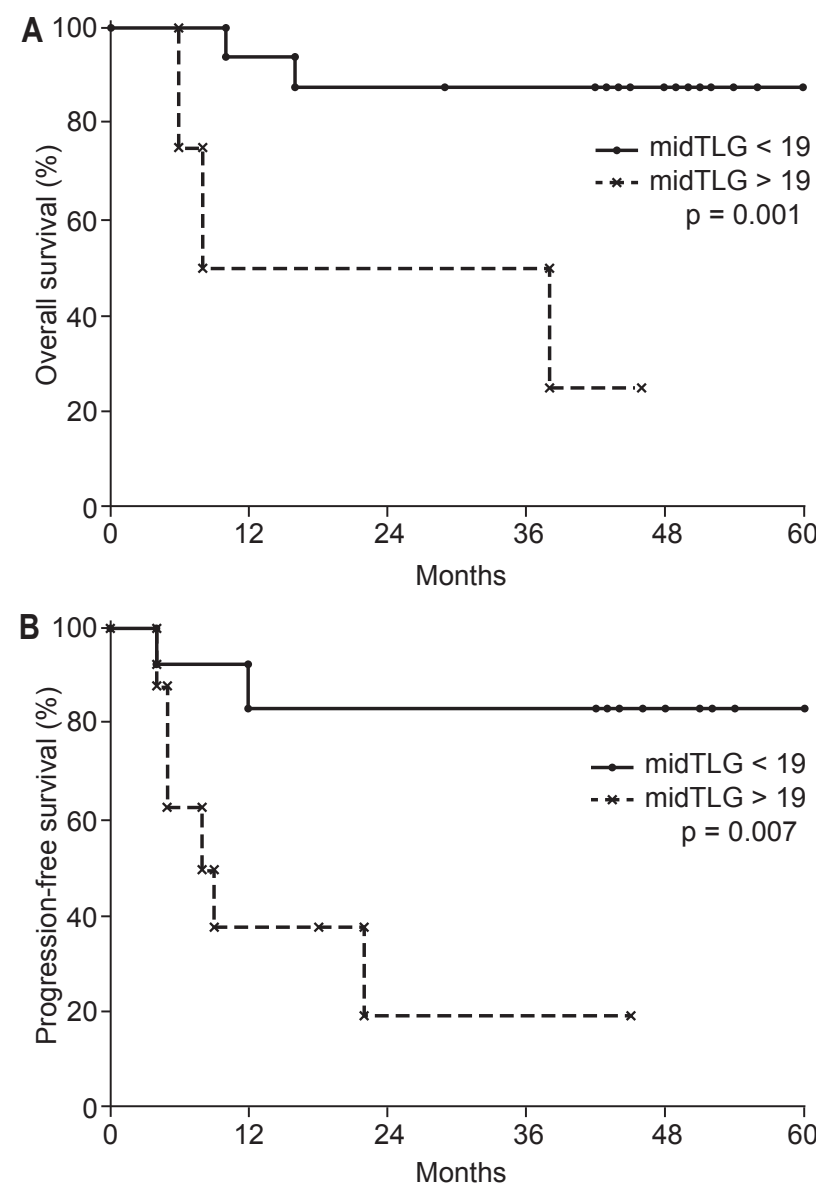

Fig. 1. Overall survival (a) and progression-free survival (b) rates according to midTLG (total lesion glycolysis of ${ }^{18} \mathrm{~F}$-fluorodeoxyglucose positron emission tomography-computed tomography [FDG PET/CT] during radiotherapy).

(3-year, $73 \%$ vs. $20 \%$, respectively; $p=0.005$ ). The 3 -year PFS rate of patients with midTLG $>19.0 \mathrm{~g}$ was $19 \%$ and the rate in patients with midTLG $<19.0 \mathrm{~g}$ was $83 \%(p=0.007)$. Table 3 shows the 3 -year OS and PFS rates according to the clinical variables and metabolic parameters. Multivariate analysis

Table 2. Comparison of PET-CT parameters before and during RT

\begin{tabular}{lccc}
\hline & Before RT & During RT & p-value \\
\hline SUV $_{\text {max }}(g / \mathrm{mL})$ & $6.9 \pm 3.8$ & $4.2 \pm 2.5$ & 0.018 \\
SUV & $4.7 \pm 2.6$ & $2.7 \pm 1.8$ & 0.009 \\
MTV $\left(\mathrm{cm}^{3}\right)$ & $5.0 \pm 2.5$ & $7.0 \pm 7.3$ & 0.080 \\
TLG $(\mathrm{g})$ & $25.8 \pm 22.3$ & $19.5 \pm 16.9$ & 0.315 \\
\hline
\end{tabular}

Values are presented as mean \pm standard deviation.

PET-CT, positron emission tomography-computed tomography; RT, radiotherapy; SUV ${ }_{\text {maxı }}$ maximum standardized uptake value; SUV meanı mean standardized uptake value; MTV, metabolic tumor volume; TLG, total lesion glycolysis.

${ }^{a}$ Using a paired t-test. 
Table 3. Univariate analysis for OS and PFS

\begin{tabular}{|c|c|c|c|c|c|}
\hline Variable & No. of patients & $3-y r$ OS (\%) & p-value & 3-yr PFS (\%) & $p$-value \\
\hline \multicolumn{6}{|l|}{ Stage } \\
\hline$\|\| I I$, & 6 & 100 & 0.544 & 63 & 0.703 \\
\hline IV & 14 & 71 & & 57 & \\
\hline \multicolumn{6}{|l|}{ T stage } \\
\hline $\mathrm{T} 1, \mathrm{~T} 2$ & 4 & 75 & 0.999 & 50 & 0.881 \\
\hline $\mathrm{T} 3, \mathrm{~T} 4$ & 16 & 81 & & 63 & \\
\hline \multicolumn{6}{|l|}{ N stage } \\
\hline No & 9 & 89 & 0.746 & 65 & 0.587 \\
\hline $\mathrm{N} 1, \mathrm{~N} 2$ & 11 & 73 & & 55 & \\
\hline \multicolumn{6}{|l|}{ Response } \\
\hline$C R, P R$ & 15 & 80 & 0.391 & 73 & $0.005^{*}$ \\
\hline SD & 5 & 80 & & 20 & \\
\hline \multicolumn{6}{|l|}{$\operatorname{preSUV}_{\max }(\mathrm{g} / \mathrm{mL})$} \\
\hline$<8.0$ & 13 & 85 & 0.168 & 62 & 0.648 \\
\hline$>8.0$ & 7 & 57 & & 57 & \\
\hline \multicolumn{6}{|l|}{ preMTV $\left(\mathrm{cm}^{3}\right)$} \\
\hline$<5.5$ & 13 & 85 & 0.168 & 69 & 0.185 \\
\hline$>5.5$ & 7 & 71 & & 43 & \\
\hline \multicolumn{6}{|l|}{ preTLG (g) } \\
\hline$<39.0$ & 16 & 88 & $0.004^{*}$ & 63 & 0.431 \\
\hline$>39.0$ & 4 & 50 & & 50 & \\
\hline \multicolumn{6}{|l|}{$\operatorname{midSUV}_{\max }(\mathrm{g} / \mathrm{mL})$} \\
\hline$<3.5$ & 10 & 90 & 0.137 & 70 & 0.477 \\
\hline$>3.5$ & 10 & 70 & & 48 & \\
\hline \multicolumn{6}{|l|}{$\operatorname{midMTV}\left(\mathrm{cm}^{3}\right)$} \\
\hline$<4.5$ & 9 & 100 & $0.022^{*}$ & 78 & 0.154 \\
\hline$>4.5$ & 11 & 64 & & 44 & \\
\hline \multicolumn{6}{|l|}{ midTLG (g) } \\
\hline$<19.0$ & 12 & 100 & $0.001^{*}$ & 83 & $0.007^{*}$ \\
\hline$>19.0$ & 8 & 50 & & 19 & \\
\hline
\end{tabular}

OS, overall survival; PFS, progression-free survival; $C R$, complete remission; PR, partial remission; SD, stable disease; PET-CT, positron

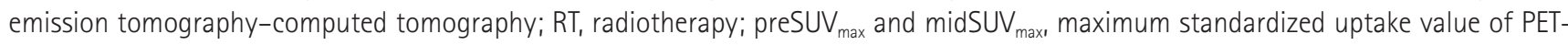
CT before and during RT; preMTV and midMTV, metabolic tumor volume of PET-CT before and during RT; preTLG and midTLG, total lesion glycolysis of PET-CT before and during RT.

${ }^{*} \mathrm{p}<0.05$.

revealed that midTLG as a continuous variable and no tumor response to RT were independent predictors of shorter PFS ( $p$ $<0.05$ ) (Table 4). MidTLG was also an independent prognostic factor for OS ( $p<0.05$ ) (Table 4). OS and PFS curves according to midTLG are illustrated in Fig. 1.

\section{Discussion and Conclusion}

Fractionated RT with concurrent chemotherapy is the standard treatment for locally advanced head and neck cancer, but it takes 6-7 weeks to complete treatment and most patients suffer from acute toxicities. If long-term treatment outcomes could be predicted during RT, the treatment plan for each individual patient could be modified. Therefore, the predictive or prognostic value of interim FDG PET/CT is currently being investigated.

FDG PET/CT is widely used for diagnosis and treatment planning in patients with head and neck cancer; however, the prognostic value of the FDG PET/CT parameters remains under investigation. MTV and TLG are volume-based metabolic parameters derived from VOI-based automated assessments. Because TLG is the product of MTV and SUV $V_{\text {mean }}$ the TLG value is a measure of both the volumetric burden and the metabolic activity of a tumor.

MTV or TLG value can be affected by the threshold of VOI. VOI was delineated with variable methods in previous studies 
Table 4. Multivariate analysis of OS and PFS

\begin{tabular}{|c|c|c|c|c|}
\hline & \multicolumn{2}{|c|}{ OS } & \multicolumn{2}{|l|}{ PFS } \\
\hline & HR (95\% Cl) & $p$-value & HR (95\% Cl) & $p$-value \\
\hline preTLG & $1.02(0.99-1.06)$ & 0.245 & $1.00(0.96-1.04)$ & 0.913 \\
\hline $\operatorname{midTLG}$ & $1.08(1.01-1.15)$ & 0.023 & $1.07(1.01-1.13)$ & 0.016 \\
\hline Response (CR, PR vs. SD) & $1.56(0.24-10.24)$ & 0.645 & 6.39 (1.28-31.88) & 0.024 \\
\hline
\end{tabular}

OS, overall survival; PFS, progression-free survival; $\mathrm{HR}$, hazard ratio; $\mathrm{Cl}$, confidence interval; PET-CT, positron emission tomographycomputed tomography; RT, radiotherapy; preTLG and midTLG, total lesion glycolysis of PET-CT before and during RT; CR, complete response; PR, partial response; SD, stable disease.

that evaluated the prognostic value of volumetric parameters of FDG PET/CT. A fixed value of SUV or percentage of SUV $V_{\text {max }}$ is used for VOI determination. A fixed SUV of 2.0-3.0 and 40\%$50 \%$ of $\mathrm{SUV}_{\max }$ were frequently used thresholds in previous studies [17] and $50 \%$ of SUV ${ }_{\max }$ was used as a threshold in the present study because it has been identified as a reasonable choice in phantom studies [15].

TLG was a significant prognostic factor for OS in patients with tonsil cancer [18]. In a study of 74 oropharyngeal cancer patients who received CCRT, both pre-treatment and midtreatment MTV were associated with OS and PFS [19]. In a meta-analysis of more than 1,100 head and neck cancer patients, both MTV and TLG of pretreatment PET/CT were prognostic factors for OS [17]. The cut-off values of studies included in that meta-analysis were $7.7-45 \mathrm{~cm}^{3}$ for MTV and 55-330 $\mathrm{g}$ for TLG. In the current study, which had a relatively small number of head and neck cancer patients, a higher TLG or MTV before RT was associated with lower OS rates. The cutoff values of our study ( $5.5 \mathrm{~cm}^{3}$ for MTV and $39.0 \mathrm{~g}$ for TLG) were lower than other studies. This is probably because onefourth of our patients underwent neoadjuvant chemotherapy before FDG PET/CT.

Min et al. [20] reported that among the parameters of SUV $_{\max }$ MTV, and TLG of PET/CT during RT, TLG was the best prognostic indicator of oncologic outcome. Also in our study, TLG during RT was the most statistically significant prognostic factor for OS and PFS. However, although the results of the two studies were similar, they used different TLG cut-off values (9.4 vs. 19.0). Differences in the study populations, timing of scanning during RT, PET/CT scanner used, image analysis software, and methods for obtaining MTV and TLG may have caused the different optimal cut-off values. Volume-based metabolic parameters such as MTV and TLG were changed time-dependently $[21,22]$. When early scanning at 60 minutes after FDG injection was compared with late scanning at 120 minutes, TLG was significantly increased at delayed phase.
Chen et al. [23] measured the SUV $\mathrm{max}_{\text {max }}$ at both the primary tumor and metastatic lymph node before and during RT. The authors demonstrated that a higher interim SUV $V_{\text {max }}$ or lower reduction ratio of the SUV $V_{\text {max }}$ at the primary tumor was a poor prognostic factor (they did not analyze MTV or TLG). Other investigators have suggested that measurements of metabolic parameters on PET/CT during RT were more prognostic at nodal sites than in the primary tumor, because they found less variability in measurements at the nodal sites [24]. Lin et al. [25] identified nodal SUV $V_{\text {mean }}$ and a reduction of nodal MTV and TLG of $>50 \%$ during RT as prognostic factors. Which one has more significant value for predicting treatment outcomes, metabolic parameters measured at primary tumor or nodal site, remains a research issue.

This study has several limitations. The small number of patients and heterogeneity of primary sites, stages of disease, and treatment methods might have resulted in unreliable cutoff value of PET/CT parameters. Previous other studies also had limitation of small sample size and heterogeneity of the population [26].

The results of our study and of these previous studies suggest that FDG PET/CT during CCRT or RT could be used to assess treatment outcomes. We acquired FDG PET/CT images in the third to fourth week of RT, but earlier assessments (i.e., 2 weeks after the start of RT) are thought to be more favorable [26]. Performing FDG PET/CT early during RT could be useful in differentiating metabolic changes from inflammatory changes and in enabling early decisions to be made regarding modification of treatment plans.

There remain many unsolved issues regarding the optimal timing of FDG PET/CT during RT, the selection of the most useful PET/CT parameters and their optimal cut-offs, and choice of the most appropriate treatment plans for patients identified as having poor prognostic factors. Thus, larger scale investigations with more a homogeneous patient group are needed. 


\section{Conflict of Interest}

No potential conflict of interest relevant to this article was reported.

\section{Acknowledgments}

This research was supported by the Basic Science Research Program through the National Research Foundation of Korea (NRF) funded by the Ministry of Science, ICT and Future Planning (No. NRF-20161A2B4012095).

\section{References}

1. Siegel RL, Miller KD, Jemal A. Cancer statistics, 2016. CA Cancer J Clin 2016;66:7-30.

2. Bourhis J, Le Maitre A, Baujat B, et al. Individual patients' data meta-analyses in head and neck cancer. Curr Opin Oncol 2007;19:188-94.

3. Bonner JA, Harari PM, Giralt J, et al. Radiotherapy plus cetuximab for squamous-cell carcinoma of the head and neck. N Engl J Med 2006;354:567-78.

4. Bonner JA, Harari PM, Giralt J, et al. Radiotherapy plus cetuximab for locoregionally advanced head and neck cancer: 5-year survival data from a phase 3 randomised trial, and relation between cetuximab-induced rash and survival. Lancet Oncol 2010;11:21-8.

5. Givens DJ, Karnell LH, Gupta AK, et al. Adverse events associated with concurrent chemoradiation therapy in patients with head and neck cancer. Arch Otolaryngol Head Neck Surg 2009;135:1209-17.

6. Garden AS, Harris J, Vokes EE, et al. Preliminary results of Radiation Therapy Oncology Group 97-03: a randomized phase ii trial of concurrent radiation and chemotherapy for advanced squamous cell carcinomas of the head and neck. J Clin Oncol 2004;22:2856-64.

7. Ang KK, Harris J, Garden AS, et al. Concomitant boost radiation plus concurrent cisplatin for advanced head and neck carcinomas: Radiation Therapy Oncology Group phase II trial 99-14. J Clin Oncol 2005;23:3008-15.

8. Syed R, Bomanji JB, Nagabhushan $N$, et al. Impact of combined (18)F-FDG PET/CT in head and neck tumours. Br J Cancer 2005;92:1046-50.

9. Baek $\mathrm{CH}$, Chung MK, Son $\mathrm{Yl}$, et al. Tumor volume assessment by $18 \mathrm{~F}-\mathrm{FDG}$ PET/CT in patients with oral cavity cancer with dental artifacts on CT or MR images. J Nucl Med 2008:49:1422-8.
10. Abgral $R$, Keromnes $N$, Robin $P$, et al. Prognostic value of volumetric parameters measured by 18 F-FDG PET/CT in patients with head and neck squamous cell carcinoma. Eur 1 Nucl Med Mol Imaging 2014;41:659-67.

11. Kim JW, Oh JS, Roh JL, et al. Prognostic significance of standardized uptake value and metabolic tumour volume on 18F-FDG PET/CT in oropharyngeal squamous cell carcinoma. Eur J Nucl Med Mol Imaging 2015;42:1353-61.

12. Abgral R, Le Roux PY, Keromnes N, et al. Early prediction of survival following induction chemotherapy with DCF (docetaxel, cisplatin, 5-fluorouracil) using FDG PET/CT imaging in patients with locally advanced head and neck squamous cell carcinoma. Eur J Nucl Med Mol Imaging 2012;39:1839-47.

13. Yoon DH, Cho Y, Kim SY, et al. Usefulness of interim FDGPET after induction chemotherapy in patients with locally advanced squamous cell carcinoma of the head and neck receiving sequential induction chemotherapy followed by concurrent chemoradiotherapy. Int J Radiat Oncol Biol Phys 2011;81:118-25.

14. Kim S, Oh SW, Kim JS, Kim KH, Kim YK. Prediction of response by FDG PET early during concurrent chemoradiotherapy for locally advanced non-small cell lung cancer. Radiat Oncol J 2014;32:231-7.

15. Ciernik IF, Dizendorf E, Baumert BG, et al. Radiation treatment planning with an integrated positron emission and computer tomography (PET/CT): a feasibility study. Int J Radiat Oncol Biol Phys 2003;57:853-63.

16. Hyun SH, Choi JY, Kim K, et al. Volume-based parameters of (18)F-fluorodeoxyglucose positron emission tomographyl computed tomography improve outcome prediction in earlystage non-small cell lung cancer after surgical resection. Ann Surg 2013;257:364-70.

17. Pak K, Cheon GJ, Nam HY, et al. Prognostic value of metabolic tumor volume and total lesion glycolysis in head and neck cancer: a systematic review and meta-analysis. J Nucl Med 2014;55:884-90.

18. Moon SH, Choi JY, Lee HJ, et al. Prognostic value of 18F-FDG $\mathrm{PET} / \mathrm{CT}$ in patients with squamous cell carcinoma of the tonsil: comparisons of volume-based metabolic parameters. Head Neck 2013;35:15-22.

19. Pollom EL, Song J, Durkee BY, et al. Prognostic value of midtreatment FDG-PET in oropharyngeal cancer. Head Neck 2016;38:1472-8.

20. Min $M$, Lin $P$, Lee $M T$, et al. Prognostic role of metabolic parameters of (18)F-FDG PET-CT scan performed during radiation therapy in locally advanced head and neck squamous cell carcinoma. Eur J Nucl Med Mol Imaging 2015;42:1984-94. 
21. Kitao $T_{1}$ Hirata $K_{1}$ Shima $K_{1}$ et al. Reproducibility and uptake time dependency of volume-based parameters on FDG-PET for lung cancer. BMC Cancer 2016;16:576.

22. Toda $K$, Torihara $A$, Nakagawa $M$, et al. Time dependency of volume-based metabolic parameters obtained by dual-timepoint TOF-PET/CT for head and neck squamous cell cancer. Int J Radiat Oncol Biol Phys 2017;99(2S):E377-E378.

23. Chen SW, Hsieh TC, Yen KY, et al. Interim FDG PET/CT for predicting the outcome in patients with head and neck cancer. Laryngoscope 2014;124:2732-8.

24. Hoang JK, Das SK, Choudhury KR, Yoo DS, Brizel DM. Using FDG-PET to measure early treatment response in head and neck squamous cell carcinoma: quantifying intrinsic variability in order to understand treatment-induced change. AJNR Am J Neuroradiol 2013;34:1428-33.

25. Lin $P$, Min $M$, Lee $M$, et al. Nodal parameters of FDG PET/ CT performed during radiotherapy for locally advanced mucosal primary head and neck squamous cell carcinoma can predict treatment outcomes: SUVmean and response rate are useful imaging biomarkers. Eur J Nucl Med Mol Imaging 2017;44:801-11.

26. Garibaldi C, Ronchi S, Cremonesi M, et al. Interim (18)F-FDG $\mathrm{PET} / \mathrm{CT}$ during chemoradiation therapy in the management of head and neck cancer patients: a systematic review. Int J Radiat Oncol Biol Phys 2017;98:555-73. 